\title{
Pomegranate-type Si/C anode with SiC taped, well-dispersed tiny Si particles for lithium-ion batteries
}

\author{
Pengfei $\mathrm{WU}^{a, b, \dagger}$, Benyang $\mathrm{SHI}^{a, \dagger}$, Huibin $\mathrm{TU}^{a}$, Changqing $\mathrm{GUO}^{a}$, \\ Anhua LIU ${ }^{a, b,{ }^{*}}$, Guan YAN ${ }^{c,}$, Zhaoju YU ${ }^{a,{ }^{*}}$ \\ ${ }^{a}$ Key Laboratory of High-Performance Ceramic Fibers of Ministry of Education, \\ College of Materials, Xiamen University, Xiamen 361005, China \\ ${ }^{b}$ Shenzhen Research Institute of Xiamen University, Shenzhen 518000, China \\ ${ }^{\mathrm{c}}$ College of Chemistry and Molecular Engineering, Peking University, Beijing 100871, China
}

Received: January 25, 2021; Revised: April 19, 2021; Accepted: May 9, 2021

(c) The Author(s) 2021.

\begin{abstract}
Severe volume expansion and inherently poor lithium ion transmission are two major problems of silicon anodes. To address these issues, we proposed a pomegranate-type $\mathrm{Si} / \mathrm{C}$ composite anode with highly dispersed tiny silicon particles as the core assisted by small amount of SiC. Skillfully exploiting the high heat from magnesiothermic reduction, $\mathrm{SiC}$ can assist the good dispersion of silicon and provide good interface compatibility and chemical stability. The silicon anchored to the carbon shell provides multipoint contact mode, that together with the carbon shell frame, significantly promoting the transfer of dual charge. Besides, the pomegranate-type microcluster structure also improves the tap density of the electrode, reduces the direct contact area between active material and electrolyte, and enhances the electrochemical performance.
\end{abstract}

Keywords: $\mathrm{Si} / \mathrm{C}$ anode; anchored $\mathrm{Si}$; $\mathrm{SiC}$ tape; magnesiothermic reduction; pomegranate like

\section{Introduction}

The rapid development of new energy industries forces the exploration of lithium ion batteries (LIBs) into the higher, faster, and safer area. The widely used graphite anodes gradually show a sign of dissatisfactory, while many alloying anodes exude a strong competitive edge $[1,2]$. Silicon is considered to be the most exciting candidate material for LIBs' anode due to its high special

\footnotetext{
$\uparrow$ Pengfei Wu and Benyang Shi contributed equally to this work.

* Corresponding authors.

E-mail: A. Liu, ahliu@xmu.edu.cn; G. Yan,0006177260@pku.edu.cn; Z.Yu, zhaojuyu@xmu.edu.cn
}

capacity, suitable discharge potential, and abundant reserves. However, full industrialization of silicon anodes still faces two fatal obstacles [3,4], as follows:

1) One is huge volume expansion caused by the steady alloying phase change and excess $\mathrm{Li}^{+}$insertion, following with the pulverization of $\mathrm{Si}$, uncontrollable interfacial reaction, and unstable formation of solid electrolyte interface layers (SEI);

2) The other is the high cost of nano-silicon from an efficiency, safe, and scalable approach.

In order to avoid the limitation influence of the first disadvantage, carbon coating and nano-scaled silicon materials are adopted to form core@shell structure, yolk@shell structure, or other ingenious framework $[5,6]$. Yolk@shell structure contains a tailorable hollow void 
for the free expansion of $\mathrm{Si}$ and an armour liked shell to protect the inner active material from the direct-contact of electrolyte, guaranteeing the structure integrity and long-life cycle. Recently, various methods have been involved in preparation of yolk@shell structure, and achieved excellent results [7-9]. However, the high specific surface area increases the interfacial reaction between electrode material and electrolyte, leading to an increase in irreversible $\mathrm{Li}^{+}$loss, a decrease in coulombic efficiency (CE) and tap density. Liu et al. [10] designed a pomegranate-inspired $\mathrm{Si} / \mathrm{C}$ anode by gathering a number of yolk@shell nano particles to form a microsized particle. This novel nano-micro secondary structure deliveries high areal capacity and ultralong cycling life.

Magnesiothermic reduction (MR) can be used to fabricate Si from very cheap silica, so it is treated as a method for scale-up Si fabrication [11]. The porosity and structure of Si can be easily controlled by changing the thermal process $[12,13]$. Not only the porous silicon [14], but also the tiny $\mathrm{Si}$ nanodots or nanoparticles were prepared by MR [15]. Unfortunately, chemically inert $\mathrm{SiC}$ is always accompanied, when the previously carbon coated silica is used as the reactant because of the local superhigh temperature. To avert the unexpected side reaction, salts with suitable melting point are often employed as the heat-absorbing medium to supply a mild reaction environment [16]. However, in some works, thin or porous $\mathrm{SiC}$ layer can also protect the inner $\mathrm{Si}$ core and provide satisfying ion transfer efficiency $[17,18]$. Therefore, the limitation of the amount of $\mathrm{SiC}$ formed during MR reaction to an acceptable level can be a proper way to stabilize the $\mathrm{Si}$ anode capacity $[19,20]$.

(a)

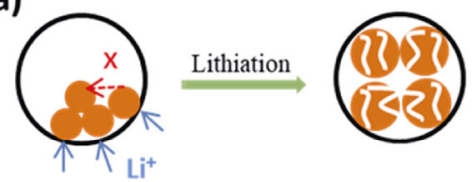

Aggregated Si
Herein, we combined the nano-micro secondary structure and MR to design a small amount SiC containing, tiny $\mathrm{Si}$ well dispersing, and pomegranate-shape-like $\mathrm{Si} / \mathrm{C}$ composite anode $(\mathrm{PSi} / \mathrm{C})$. The small amount $\mathrm{SiC}$, gained from the local high temperature of MR by controlling $\mathrm{NaCl}$, can play a role like double side tape to anchor the $\mathrm{Si}$ particles on the $\mathrm{C}$ shell with a good dispersion. This design of tape-SiC exhibits three kinds of excellent peculiarities, as follows:

1) Intrinsic chemical inert can protect $\mathrm{Si}$ from the corrosion of the permeable electrolyte.

2) $\mathrm{C}-\mathrm{SiC}-\mathrm{Si}$ interface provides better interface compatibility than $\mathrm{C}-\mathrm{Si}$.

3) Random anchored Si tiny particles on C shell supply a multipoint contact mode [21], facilitating effective $\mathrm{Li}^{+} / \mathrm{e}^{-}$transfer pathway and decreasing the diffusion length of $\mathrm{Li}^{+}$instead of various disadvantages of nanoparticles' aggregation (Figs. 1(a) and 1(b)).

What is more, the pomegranate-shape-like structure armed with carbon shell can:

1) limit the direct contact of electrolyte and silicon;

2) improve the electronic conductivity of Si nanoparticles;

3) provide hollow void for the free volume expansion of silicon.

\section{Experimental section}

\section{1 Synthesis of active materials}

\subsubsection{Synthesis of $\mathrm{SiO}_{2}$ particles}

First, $90 \mathrm{~mL}$ of ethanol (analytically pure, XILONG

(b)

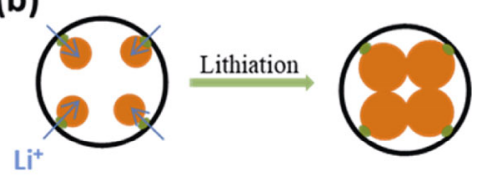

Anchored Si

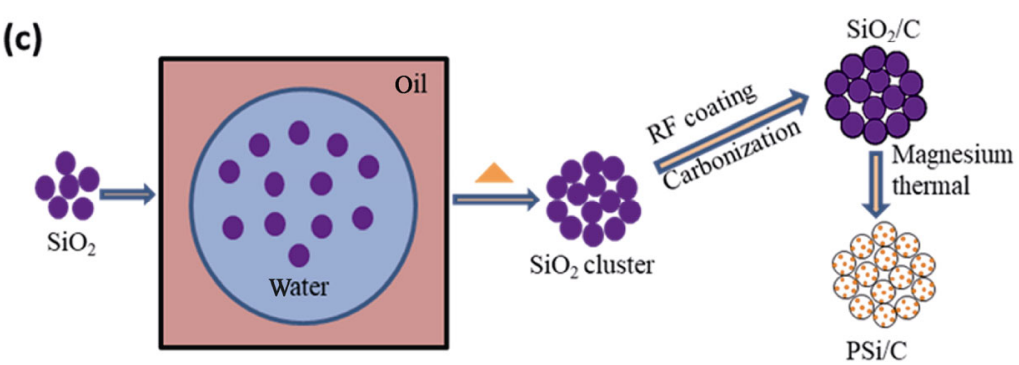

Fig. 1 Schematic illustration of lithium ion transfer pathways and the morphology evolutions of Si particles. (a) Yolk@shell structure with aggregated silicon core; (b) yolk@shell structure with anchored silicon core; (c) schematic preparation procedure of PSi/C nanocomposites. 
Scientific, China) and $9 \mathrm{~mL}$ of deionized water was added to the flask and mixed well, and then $3 \mathrm{~mL}$ of ethyl orthosilicate (analytically pure, XILONG Scientific, China) was added and heated slowly to $50{ }^{\circ} \mathrm{C}$ in an oil bath. Then, $7.5 \mathrm{~mL}$ of ammonia water (analytically pure, XILONG Scientific) was quickly added to adjust the $\mathrm{pH}$ of the solution, continuing to stir for $2 \mathrm{~h}$, and then ethanol was used for centrifugal purification three times. Finally, it was placed in a vacuum oven at $50{ }^{\circ} \mathrm{C}$ for $5 \mathrm{~h}$ to obtain $\mathrm{SiO}_{2}$ particles [22]. After the nano-scale $\mathrm{SiO}_{2}$ particles were obtained, the porous $\mathrm{Si} / \mathrm{C}$ composite materials were synthesized through the following steps.

\subsubsection{Synthesis of $\mathrm{SiO}_{2}$ micron clusters}

$0.36 \mathrm{~g}$ of $\mathrm{SiO}_{2}$ nanoparticles were uniformly dispersed into $9 \mathrm{~mL}$ of deionized water by ultrasonic dispersion, and then mixed with $72 \mathrm{~mL}$ of 1-octadecene (analytically pure, XILONG Scientific, China) solution containing $0.4 \%$ emulsifier (Hypermer 2524-NV-LQ-(AP), Croda). The mixed solution was dispersed at a high speed at $12,000 \mathrm{rpm}$ for $10 \mathrm{~min}$, and then heated in an oil bath at 96-98 ${ }^{\circ} \mathrm{C}$ for $8 \mathrm{~h}$ to evaporate the deionized water. The obtained sample was centrifuged and cleaned with $n$-hexane for three times and then calcined in the air at $550{ }^{\circ} \mathrm{C}$ for $1 \mathrm{~h}$ to remove the organic emulsifier and obtain $\mathrm{SiO}_{2}$ micron clusters.

\subsubsection{Coating of carbon layer on the surface of $\mathrm{SiO}_{2}$ micron clusters}

$0.3 \mathrm{~g}$ of $\mathrm{SiO}_{2}$ micron clusters were dispersed in $90 \mathrm{~mL}$ of deionized water, and then $3 \mathrm{~mL}$ of CTAB (analytically pure, SINOPHARM, China) and $0.3 \mathrm{~mL}$ of ammonia were added, and then stirred for $30 \mathrm{~min}$ to mix well. Next, $0.12 \mathrm{~g}$ of resorcinol and $168 \mu \mathrm{L}$ of formaldehyde solution (analytically pure, XILONG Scientific, China) were introduced, and stirred at room temperature for $16 \mathrm{~h}$. The phenolic resin-coated $\mathrm{SiO}_{2}$ micron clusters were collected by centrifugation and washed with ethanol three times. Then the powder was heated to $850{ }^{\circ} \mathrm{C}$ at a heating rate of $5{ }^{\circ} \mathrm{C} \cdot \mathrm{min}^{-1}$ in an argon atmosphere and kept for $2 \mathrm{~h}$. The final sample was named $\mathrm{SiO}_{2} / \mathrm{C}$ micron clusters.

\subsubsection{Synthesis of pomegranate-type Si/C composite}

$0.3 \mathrm{~g}$ of $\mathrm{SiO}_{2} / \mathrm{C}$ powder and $0.3 \mathrm{~g}$ of magnesium powder (analytically pure, Aladdin, China) were mixed and grounded in a mortar, and then $3 \mathrm{~g}$ of sodium chloride powder (analytically pure, XILONG Scientific, China) was added and mixed uniformly. The obtained mixture was placed in a closed crucible, and heated to $660{ }^{\circ} \mathrm{C}$ at a heating rate of $5{ }^{\circ} \mathrm{C} \cdot \mathrm{min}^{-1}$ in a tube furnace filled with argon and keep it for $4 \mathrm{~h}$ [23]. The sample powder was transferred into a $1 \mathrm{~mol} \cdot \mathrm{L}^{-1}$ dilute hydrochloric acid (analytically pure, XILONG Scientific, China) solution and stirred for $4 \mathrm{~h}$ to remove unreacted magnesium powder and by-products $\mathrm{MgO}$ and $\mathrm{Mg}_{2} \mathrm{SiO}_{4}$, while dissolving and removing $\mathrm{NaCl}$ acting as an endothermic. Finally, the product was cleaned in a $5 \mathrm{wt} \%$ HF dilute solution (analytically pure, XILONG Scientific, China) for $5 \mathrm{~min}$ to remove unreacted $\mathrm{SiO}_{2}$ in the composite material. The porous $\mathrm{Si} / \mathrm{C}$ composite material was collected by centrifugation and placed in a vacuum oven at $50{ }^{\circ} \mathrm{C}$ for $5 \mathrm{~h}$ to obtain a three-dimensional continuous porous silicon-carbon composite material $\mathrm{PSi} / \mathrm{C}$ (yield is 58\%).

\section{2 Characterization}

The morphology of the samples was characterized by scanning electron microscope (SEM, Hitachi SU-70, Japan) and transmission electron microscope (TEM, JEM-2100, Japan). The crystal structure of the products was determined using X-ray diffraction (XRD, Bruker-axs, $\mathrm{Cu} \mathrm{K} \alpha$ radiation, $40 \mathrm{kV}, 40 \mathrm{~mA}$ ). Raman spectra were obtained on HORIBA and CCD spectroscopy systems with an excitation wavelength of $532 \mathrm{~nm}$. The $\mathrm{N}_{2}$ adsorption/desorption measurement (BET, Micromeritics Tristar II 3020 static volumetric analyzer, USA) was used to identify the surface area and pore size distribution of the sample.

\section{3 Electrochemical measurements}

The electrochemical measurements were conducted by assembling 2025 coin-type half-cells in an argon-filled glove box (MBRAUN, $\mathrm{H}_{2} \mathrm{O}<0.1 \mathrm{ppm}, \mathrm{O}_{2}<0.1 \mathrm{ppm}$ ) with lithium metal (analytically pure, Canrd, China) as the counter/reference electrode and Celgard 2400 (analytically pure, DoDoChem, China) as the separator. The anode was prepared by using deionized water as a solvent and using active materials, conducting agent (Super C, analytically pure, DoDoChem, China), and sodium carboxymethyl cellulose (CMC, analytically pure, DoDoChem, China) at a mass ratio of $6: 2: 2$. The electrodes were prepared by coating the slurry on copper foil at a thickness of $100 \mu \mathrm{m}$, which was then dried at $50{ }^{\circ} \mathrm{C}$ for $5 \mathrm{~h}$ and subsequent $80{ }^{\circ} \mathrm{C}$ for $12 \mathrm{~h}$ in a vacuum oven. The electrolyte (analytically pure, DoDoChem, China) was $1.0 \mathrm{M} \mathrm{LiPF}_{6}$ in $1: 1$ (weight 
ratio) ethylene carbonate (EC)/diethylene carbonate (DEC), with $10 \mathrm{wt} \%$ fluoroethylene carbonate (FEC) additive. All batteries were tested between 2.0 and $0.01 \mathrm{~V}$ using the Neware battery test system. Electrochemical impedance curve (EIS) was obtained in the frequency range of $100 \mathrm{kHz}$ to $0.01 \mathrm{~Hz}$, and cyclic voltammetry (CV) measurements were performed at a scan rate of $0.05 \mathrm{mV} \cdot \mathrm{s}^{-1}$ at a voltage range of $0.01-2 \mathrm{~V}$.

\section{Results and discussion}

The $\mathrm{SiO}_{2}$ nano-particles synthesized from the hydrolyzation of tetraethyl orthosilicate (TEOS) present regular sphere shape with smooth surface and uniform size $(\sim 180 \mathrm{~nm})$ (Fig. S1 in the Electronic Supplementary Material (ESM)). In order to gain the micro-sized cluster particles, aqueous dispersion of silica particles is dropped into 1-octadecene containing $0.04 \mathrm{wt} \%$ Hypermer as emulgator under highspeed stirring. Carbon layer is formed from the normal coating and carbonization of resorcinol formaldehyde (RF) resin. After MR procedure and etching of side products, the final $\mathrm{PSi} / \mathrm{C}$ particles are prepared. The intuitive steps are shown in Fig. 1(c).

Figure 2 shows the SEM images of $\mathrm{SiO}_{2}$ microclusters, $\mathrm{SiO}_{2} / \mathrm{C}$ and $\mathrm{PSi} / \mathrm{C}$ composite materials. It can be seen from Fig. 2(a) that the size of the $\mathrm{SiO}_{2}$ micron clusters synthesized by the microemulsion method is in the range of $1-4 \mu \mathrm{m}$, and the micro-sized electrode material can greatly increase the tap density and volume capacity of the battery, effectively avoiding the defects caused
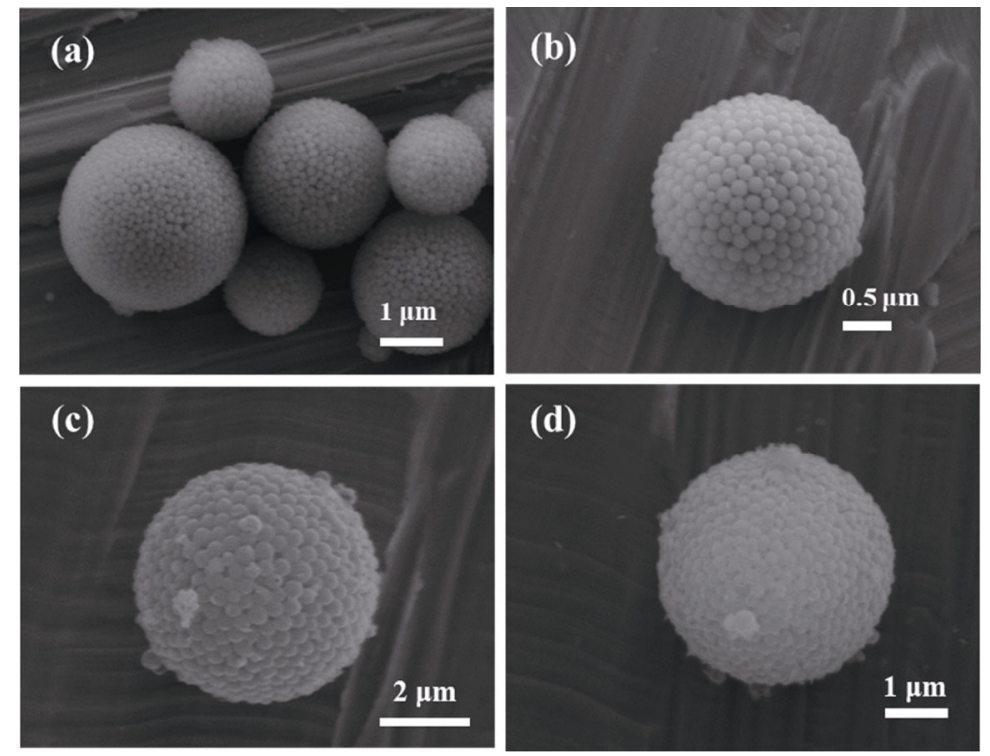

Fig. 2 Morphological characterization by SEM. (a) Micro-sized $\mathrm{SiO}_{2}$ cluster particles. (b) Single micro-sized $\mathrm{SiO}_{2}$ cluster particle. (c) Micro-sized $\mathrm{SiO}_{2} / \mathrm{C}$ cluster particles. (d) Pomegranate-shape-like PSi/C nanocomposites. 
transfer ability. $\mathrm{No} \mathrm{SiO}_{2}$ is observed in the SEM image of the PSi/C composite, and the outer carbon shell can maintain a perfect spherical shape. The three-dimensional interconnected carbon shell can effectively isolate the direct contact between silicon and electrolyte, thereby reducing the formation of SEI film and inhibiting the continuous consumption of electrolyte, which can greatly improve the coulombic efficiency of the battery.

In order to more intuitively observe the composition and structure changes of these composite materials during the entire preparation process, we performed SEM-EDS mapping analysis on $\mathrm{SiO}_{2}$ microclusters, $\mathrm{SiO}_{2} / \mathrm{C}$ and PSi/C composite materials, shown in Fig. S2 in the ESM. As can be seen from Fig. S2(a) in the ESM, only $\mathrm{Si}$ (red) and $\mathrm{O}$ (yellow) are two elements in the $\mathrm{SiO}_{2}$ micron clusters before coating the amorphous carbon layer, and they are evenly distributed. The $\mathrm{Si}, \mathrm{C}$ (blue), and $\mathrm{O}$ elements in the $\mathrm{SiO}_{2} / \mathrm{C}$ composite material are evenly distributed, indicating the presence of $\mathrm{SiO}_{2}$ and amorphous carbon in the material (Fig. S2(b) in the ESM). After MR and etching, the content of $\mathrm{O}$ element in $\mathrm{PSi} / \mathrm{C}$ composites is greatly reduced. While the $\mathrm{Si}$ and $\mathrm{C}$ elements can still be evenly distributed in the sample. This shows that MR process causes $\mathrm{SiO}_{2}$ to be partially reduced to elemental $\mathrm{Si}$, which is also the same as observed in the above SEM images. The silicon produced by this inexpensive method has a tiny size which limits the damage caused by the volume expansion of silicon during the cycle $[25,26]$.

The internal structure and morphology of the PSi/C composite were further characterized by TEM, and the results are shown in Fig. 3. After the MR step and acid etching, unreacted $\mathrm{Mg}$ and undesirable products $\mathrm{MgO}$ are removed, leaving well dispersed tiny sized $\mathrm{Si}$ nanoparticles within $10 \mathrm{~nm}$ (Figs. 3(a) and 3(b)). As seen in the SEM image, the PSi/C composite presents a relatively regular spherical shape with a size of about $4 \mu \mathrm{m}$, and a large number of amorphous carbon shells and pores can be observed. It can be seen from the enlarged view in Fig. 3(b) that the size of the spherical carbon shell is consistent with the size of $\mathrm{SiO}_{2}$ synthesized by the sol-gel method (about $180 \mathrm{~nm}$ ), which indicates that the thin amorphous carbon layer is successfully coated on the $\mathrm{SiO}_{2}$ nanoparticles' surface. The carbon layer (thickness of about $8 \mathrm{~nm}$ ) can not only isolate the direct contact between the internal silicon particles and the electrolyte, but also provide with better mechanical stability, cushion the silicon volume expansion in the process of charge and discharge, and improve the cycling stability of the electrode material [27].

From the HRTEM images in Figs. 3(c) and 3(d), a lattice fringe of $0.31 \mathrm{~nm}$ can be observed (white areas), corresponding to the (111) plane of crystalline silicon, which also proves that $\mathrm{SiO}_{2}$ is transformed into crystalline $\mathrm{Si}$ after the MR procedure. Because every Si tiny particle is isolated with each other, the obtained silicon is not
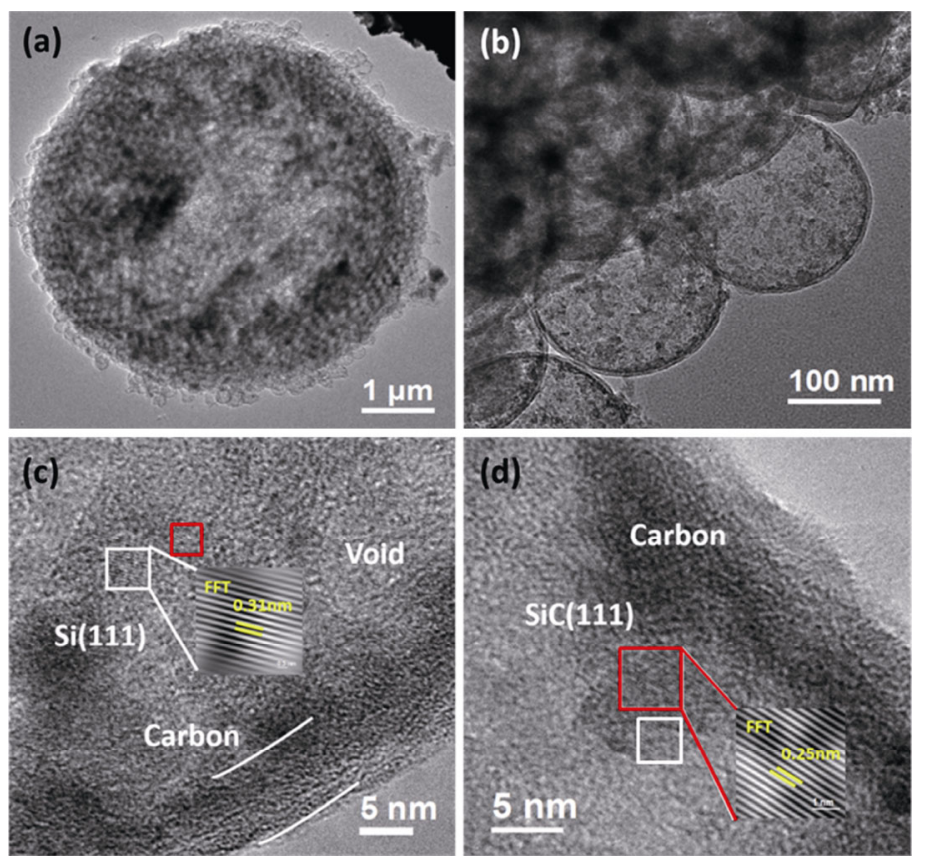

Fig. 3 Morphological and structural characterization of PSi/C composite by TEM. (a) Single PSi/C particle; (b) enlarge area vision; (c) and (d) HRTEM images of different areas in one particle. The white area is Si and the red area is SiC. 
the connected porous Si material but the well dispersed tiny $\mathrm{Si}$ nanoparticles which is different from the most MR related works. Importantly, the reason that these $\mathrm{Si}$ nanoparticles can be well dispersed or anchored on the carbon inner wall after such harsh thermal reaction is unsettled, although Chen et al. [28] also proposed a yolk@shell structure with tiny Si dispersed inside by MR method. The results show that the material with different length $(0.25 \mathrm{~nm})$ lattice fringe adjacent to these $\mathrm{Si}$ particles is $\mathrm{SiC}$ [29]. It is well known that the temperature of the direct reaction between $\mathrm{Si}$ and $\mathrm{C}$ will only occur when the temperature exceeds $1100{ }^{\circ} \mathrm{C}$, which is much higher than the temperature used for the MR reduction. In this study, $\mathrm{NaCl}$ cannot fully absorb the large amount of heat released by the thermal reaction, heating the surrounding atmosphere to the temperature required for the reaction between $\mathrm{Si}$ and $\mathrm{C}$, thereby forming SiC nanodots [30]. However, the heat formed from MR reaction is hardly controlled, so the size of $\mathrm{SiC}$ and the size ratio of $\mathrm{SiC}$ with $\mathrm{Si}$ in one particle are irregular. The adjacent relationship between $\mathrm{SiC}$ and $\mathrm{Si}$ endows $\mathrm{SiC}$ an interesting role as double side tape to anchor the Si particles onto the $\mathrm{C}$ inner wall, providing a good compatible interface. Compared with amorphous carbon materials, it has better mechanical stability, so a small amount of SiC can increase the structural stability of electrode materials [31-33].

From the selected area electron diffraction pattern of the composite material, it can also be seen that there is a crystal structure (Fig. S3(a) in the ESM), which also corresponds to the above-mentioned $\mathrm{Si}$ and $\mathrm{SiC}$ crystals. Figure S3(b) in the ESM shows the carbon skeleton after $\mathrm{Si}$ is etched away with a dilute $\mathrm{NaOH}$ solution. The existence of three-dimensional interconnected amorphous carbon shells can be undoubtly seen and they all show a relatively regular spherical shape, which indicates that resorcinol and formaldehyde have penetrated into the material and covered the surface of each $\mathrm{SiO}_{2}$. The carbon shell can still remain intact during the subsequent MR process.

The X-ray diffraction (XRD) pattern of the PSi/C composite material can reflect the composition and phase structure of the material, as shown in Fig. 4(a). It can be detected that the characteristic peaks of $2 \theta$ at $28.4^{\circ}, 47.3^{\circ}, 56.1^{\circ}, 69.1^{\circ}$, and $76.4^{\circ}$ correspond to the (111), (220), (311), (440), and (331) planes of Si (JPCDS No. 27-1402), respectively, which shows that $\mathrm{SiO}_{2}$ reacts with magnesium vapor to form $\mathrm{Si}$ during the thermal reduction [33]. The characteristic peak of $2 \theta$ at $35.6^{\circ}$ corresponds to the (111) plane of SiC (JPCDS No. 74-2307), which means that the local heat generated during MR causes the formation of $\mathrm{SiC}$ due to the direct
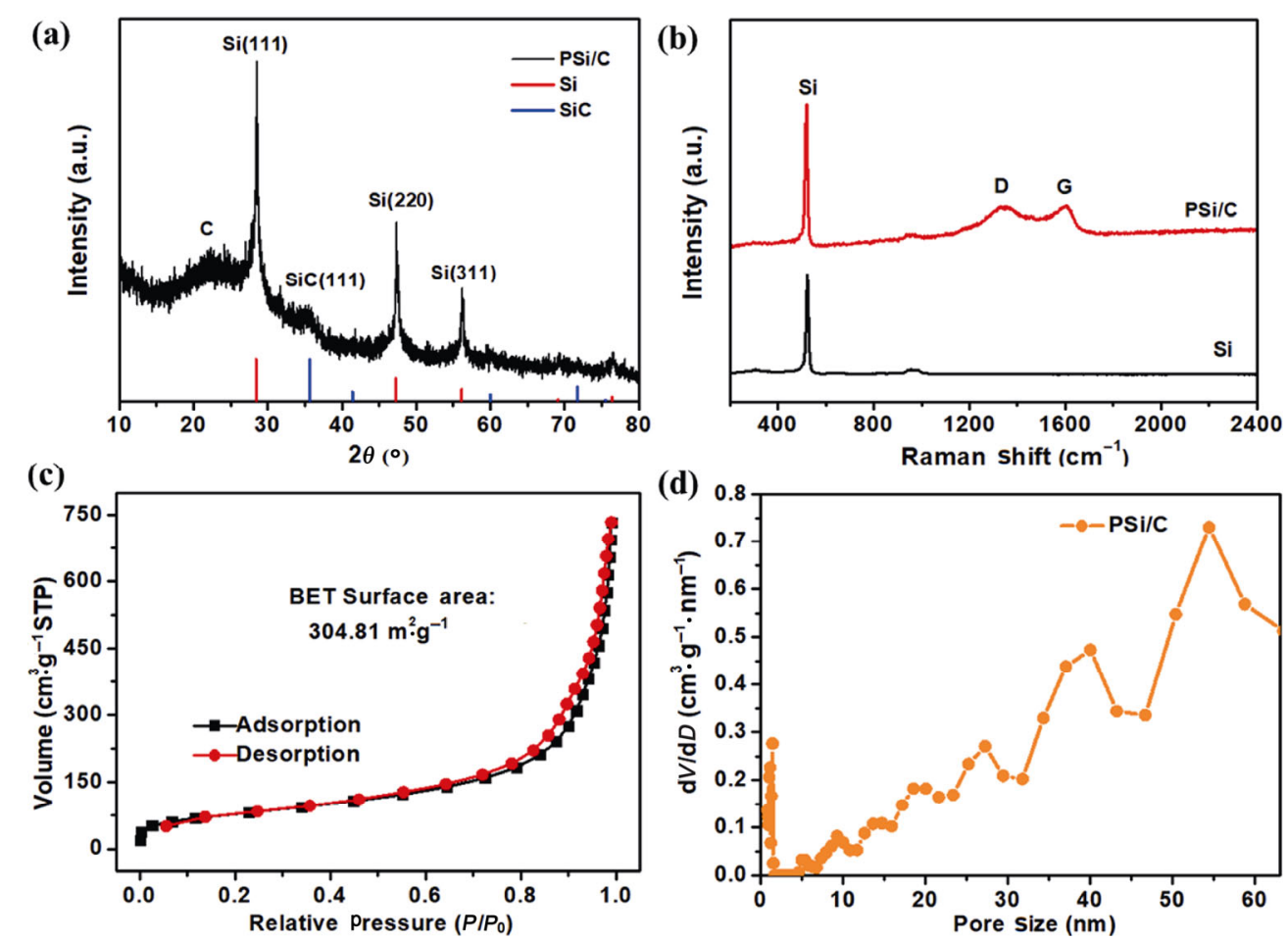

Fig. 4 Characterization of PSi/C composite. (a) XRD pattern; (b) Raman spectra; (c) $\mathrm{N}_{2}$ adsorption-desorption curve; (d) pore size distribution curve. 
react of Si and amorphous carbon [20]. The broad peak around $22^{\circ}$ can be attributed to the presence of the amorphous carbon layer, except that no other impurity peaks were found. These results are consistent with those observed in the above TEM images.

The Raman curve of the PSi/C powder in Fig. 4(b) shows two characteristic peaks of carbon at 1337.8 and $1598.6 \mathrm{~cm}^{-1}$, corresponding to the disordered $\mathrm{D}$ band and graphite $\mathrm{G}$ band $\left(I_{\mathrm{D}} / I_{\mathrm{G}}=1.09\right)$. The strong $\mathrm{D}$ peak indicates that the carbon material in the composite material is in the state of amorphous carbon, which is consistent with the above XRD pattern. The two peaks centered at 516 and $948 \mathrm{~cm}^{-1}$ correspond to the characteristic peaks of crystalline silicon. The wavelength of $516 \mathrm{~cm}^{-1}$ is lower than that of bulk silicon $\left(520 \mathrm{~cm}^{-1}\right)$, which means its tiny nano-sized morphology $[34,35]$. The Raman result is consistent with the observation in HRTEM.

The porous properties of $\mathrm{PSi} / \mathrm{C}$ composites were measured by $\mathrm{N}_{2}$ adsorption-desorption isotherm. Figure 4(c) shows a typical type IV adsorption-desorption isotherm, which proves the porous characteristics of the sample [36,37], and the BET specific surface area (SSA) is $304.81 \mathrm{~m}^{2} \cdot \mathrm{g}^{-1}$. The reason of high SSA is mainly attributed to the amorphous carbon layer, the internal unoccupied inner hollow space, and the voids/channels formed during MR. Barrete-Joynere-Halenda (BJH) method is used to calculate the pore size distribution of the sample (Fig. 4(d)). The results show that the pore structure of the sample covers the micro- and meso-pores. The smaller pores are left by the removal of surfactants Hypermer and CTAB, while the larger pores are formed from MR and acid washing process, or the space gap between different Si nanoparticles. These pores provide a buffer space for the volume expansion in the lithium process and give the sample a good cycling stability.

According to the XPS survey vision of the PSi/C composite, there are three elements $(\mathrm{O}, \mathrm{C}$, and $\mathrm{Si})$ in the as-characterized sample without any other heteroatoms (Fig. S4(a) in the ESM). After fitting the high resolution vision of Si 2p (Fig. S4(b) in the ESM), two peaks representing $\mathrm{Si}-\mathrm{O}$ and $\mathrm{Si}-\mathrm{C}$ bonds can be recognized at 103.7 and $104.5 \mathrm{eV}$, respectively. The former $\mathrm{Si}-\mathrm{O}$ band should be introduced by the absorption of water or oxygen on the surface of nano-Si particles because of the high surface energy of nano-scaled Si materials [38]. And the peak appearing at $99-103 \mathrm{eV}$ is assigned to $\mathrm{Si}$ nanoparticles. However, XPS is a method to analyze the elements on the surface of the sample, so the ratio can not reflect the real value effectively. In order to determine the correct proportions of free carbon, $\mathrm{SiC}$, and $\mathrm{Si}$ in this composite, we resort to TGA and $\mathrm{C} / \mathrm{S}$ analyzer and the results are shown in Fig. S5 and Table S1 in the ESM. The content of free carbon can be easily gained from TGA (18.6\%), and the total C element in the sample is $25.9 \%$ from $\mathrm{C} / \mathrm{S}$ analyzer. Finally, the proportions of different components are calculated as $18.6 \%, 24.3 \%$, and $57.1 \%$ of free carbon, $\mathrm{SiC}$, and $\mathrm{Si}$, respectively.

$\mathrm{PSi} / \mathrm{C}$ was coated on the $\mathrm{Cu}$ foil cooperated with binder and conductive agent as the anode (surface density is $3.49 \mathrm{mg} \cdot \mathrm{cm}^{-3}$ ), and its electrochemical properties were tested in a half cell. In order to better analyze the redox reaction of the anode material during the cycle, the $\mathrm{CV}$ curve of the PSi/C composite material was tested at a scan rate of $0.5 \mathrm{mV} \cdot \mathrm{s}^{-1}$ in the voltage range of $0.01-2.0 \mathrm{~V}$, shown in Fig. 5(a). In the first cathodic scan, there was a cathode peak at about $0.40 \mathrm{~V}$, which was mainly due to the formation of SEI film on the surface of carbon materials and the irreversible consumption of lithium
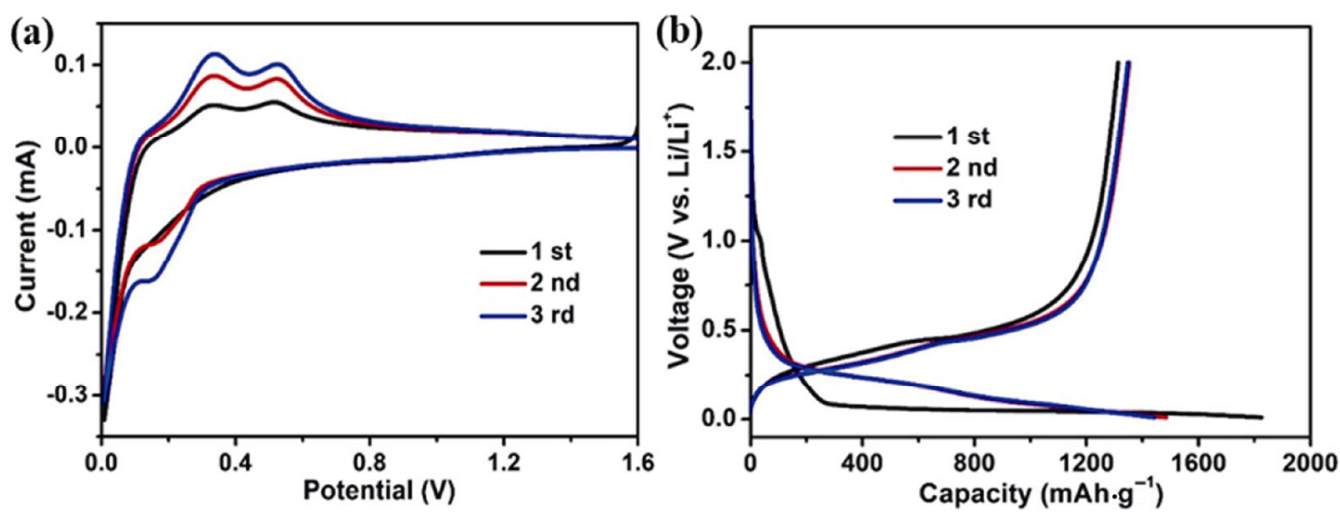

Fig. 5 Electrochemical performance of the PSi/C anode for the first 3 cycles. (a) Cyclic voltammetry curve, where the scan rate is $0.5 \mathrm{mV} \cdot \mathrm{s}^{-1}$ tested in the voltage range of $0.01-2.0 \mathrm{~V}$; (b) discharge/charge curves under a current density of $0.2 \mathrm{~A} \cdot \mathrm{g}^{-1}$. 
ions in the first lithiation process. However, the cathode peak disappeared during the subsequent cycles, and a new cathode peak appeared near $0.19 \mathrm{~V}$, which corresponds to the phase transition from amorphous a-Si to amorphous $\mathrm{a}-\mathrm{Li} \mathrm{i}_{x} \mathrm{Si}$ [39]. In the anode scan, due to the delithiation reaction of the $\mathrm{a}-\mathrm{Li}_{x} \mathrm{Si}$ alloy phase and the formation of amorphous silicon, the electrode material has two anode peaks near 0.32 and $0.53 \mathrm{~V}$. By comparing the scans of the first 3 cycles, it can be found that both the cathode peak and the anode peak increase a lot, which is mainly due to the continuous activation of the active material during the previous cycles [40].

Figure 5(b) depicts the first 3 charge and discharge characteristics of the PSi/C anode at a current density

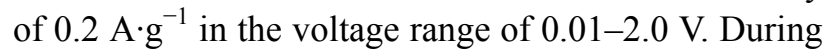
the first discharge, the longer sloping potential plateau from about $0.4 \mathrm{~V}$ is due to the irreversible formation of the SEI film, which is consistent with the results observed in the CV curve. During the first charge and discharge, the charge and discharge capacities of the PSi/C anode are $1825.8 \mathrm{mAh} \cdot \mathrm{g}^{-1}$ (energy density is $155.2 \mathrm{Wh} \cdot \mathrm{kg}^{-1}$ ) and $1313.3 \mathrm{mAh} \cdot \mathrm{g}^{-1}$, respectively, and the initial coulombic efficiency reaches $72 \%$. The initial irreversible capacity loss $(28 \%)$ was mainly attributed to the formation of the SEI film or the irreversible trap of $\mathrm{Li}^{+}$on the surface of the amorphous carbon layer [41]. In the first four deep cycles, the composites are gradually activated to form stable SEI and develop proper structural network [42]. After activation, coulombic efficiency can achieve to $97 \%$ and be stable at $99 \%$ in the future cycling (Fig. 6(b)).

In order to explore the lithium ion and charge transfer resistance of the electrode material, an EIS test was performed on the $\mathrm{PSi} / \mathrm{C}$ anode in the frequency range of $0.01-100 \mathrm{~Hz}$, and the results are shown in Figs. 6(a) and S6 in the ESM. It can be seen that the charge transfer resistance in the high-frequency region is small (about $44.57 \Omega$ ), while the slope is large in the lowfrequency region, indicating that the diffusion rate of lithium ions inside the material is high. For comparison, EIS spectrum of bare Si nanoparticles was also detected, showing a larger charge transfer resistance. The results indicate the multipoint contact mode nature between $\mathrm{Si}$ and $\mathrm{C}$ shell of the PSi/C anode material diffusion.

We tested the rate performance of $\mathrm{PSi} / \mathrm{C}$ material, and the results are shown in Fig. 6(c). The capacity of $\mathrm{PSi} / \mathrm{C}$ electrode material is about $1400,1050,800$, and $500 \mathrm{mAh} \cdot \mathrm{g}^{-1}$ at current densities of $0.2,0.5,1$, and $2 \mathrm{~A} \cdot \mathrm{g}^{-1}$, respectively. When the current density returns to $0.5 \mathrm{~A} \cdot \mathrm{g}^{-1}$
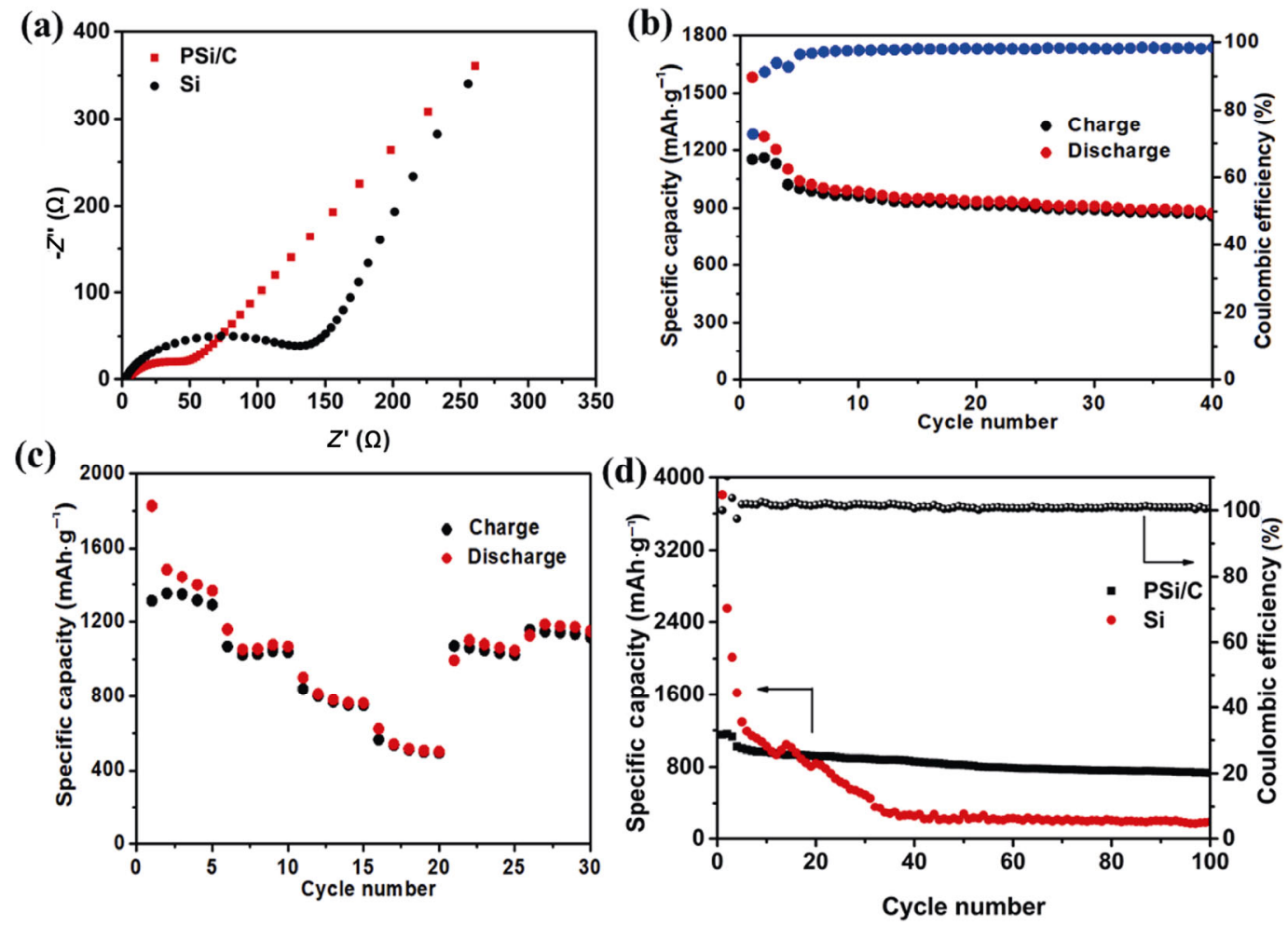

Fig. 6 Battery performance of PSi/C anode. (a) EIS curve before cycling; (b) cycling performance at $0.2 \mathrm{~A} \cdot \mathrm{g}^{-1}$ current density; (c) rate performance under various current densities; (d) cycle performance under the current density of $1 \mathrm{~A} \cdot \mathrm{g}^{-1}$ (the current density of the first 3 cycles is $0.2 \mathrm{~A} \cdot \mathrm{g}^{-1}$ ). 
again, the capacity can be restored to $1050 \mathrm{mAh} \cdot \mathrm{g}^{-1}$ with $100 \%$ recover capacity, which shows that the structure can remain stable even at a higher current density. The sufficient void space inside the PSi/C composite material can buffer the expansion of the silicon electrode, and the presence of amorphous carbon [43] and $\mathrm{SiC}$ can isolate the direct contact between silicon and the electrolyte and show good structural stability.

Figure 6(b) shows the cycle curve of the PSi/C anode at a current density of $0.2 \mathrm{~A} \cdot \mathrm{g}^{-1}$. It can be seen that the specific capacity after 40 cycles can reach $872.6 \mathrm{mAh} \cdot \mathrm{g}^{-1}$, which is about 3 times that of the graphite anode. The cycle stability at higher current density also determines an important factor in the industrialization of electrode materials. The cycle performance of the $\mathrm{PSi} / \mathrm{C}$ anode at a current density of $1 \mathrm{~A} \cdot \mathrm{g}^{-1}$ is shown in Fig. 6(d). The first 3 cycles show the activated cycles at a rate of $0.2 \mathrm{~A} \cdot \mathrm{g}^{-1}$. From the 4th to the 100th cycle, the specific capacity is reduced from 967 to $629 \mathrm{mAh} \cdot \mathrm{g}^{-1}$, more than $65 \%$ of the capacity is retained, and the coulombic efficiency is as high as $99 \%$. Si nanoparticle shows a great decrease within first 30 cycles, and it reaches almost $20 \mathrm{mAh} \cdot \mathrm{g}^{-1}$ after 40 cycles even at a small current intensity of $0.5 \mathrm{~A} \cdot \mathrm{g}^{-1}$, indicating that the $\mathrm{Si} / \mathrm{C}$ anode with special structure formed from MR reaction has good cycle stability.

Recent work related to $\mathrm{SiC}$ containing Si-based anodes for LIBs was summarized in Table S2 in the ESM to give a comparison. The pomegranate-type $\mathrm{Si} / \mathrm{C}$ composite in this work can deliver good electrochemical performance and cycling performance, and it has to be considered as an efficient method to prepare potential LIB anodes. Structure stability is the basic of cycling stability. We checked whether the pomegranate-type structure can be maintained after deep cycling by SEM characterization. From Fig. S7 in the ESM, a pomegranate-type framework can be seen clearly, suggesting that the structure can be destroyed by cycling, and the rougher surface compared with SEM image before cycling is due to uniform SEI film formed on the material surface [44]. The intact structure inhibits the continuous electrolyte deposition and ensures the uniform formation of SEI film. These factors ensure its good electrochemical performance.

\section{Conclusions}

In this work, we used low-cost TEOS as the silicon source, and synthesized a pomegranate-shape-like micron-scale
$\mathrm{Si} / \mathrm{C}$ composite through the industrially commonly used microemulsion method and magnesiothermic reduction method. In this unique structure, the sufficient void space can buffer the volume expansion of silicon during the cycle, and the well dispersed tiny silicon particles are well covered by the carbon layer to prevent excessive SEI film formation. The multipoint contact mode endowed by tape-role-like $\mathrm{SiC}$ and the three-dimensional interconnected carbon shell provide a certain path for the transmission of lithium ions and electrons, which enables the electrode material to have higher coulombic efficiency. The formation of $\mathrm{SiC}$ at local irregular high temperature by MR has an important contribution to the structural stability. Therefore, the $\mathrm{PSi} / \mathrm{C}$ anode shows better cycle stability during the cycling, and more than $65 \%$ of the capacity remains after 100 cycles at a current density of $1 \mathrm{~A} \cdot \mathrm{g}^{-1}$.

\section{Acknowledgements}

We thank the Shenzhen Science and Technology Projects (No. JCYJ20180306172957494) and National Natural Science Foundation of China (No. 5187224) for financial support.

\section{Electronic Supplementary Material}

Supplementary material is available in the online version of this article at https://doi.org/10.1007/s40145-021-0498-6.

\section{References}

[1] Ding Y, Cano Z, Yu A, et al. Automotive Li-ion batteries: Current status and future perspectives. Electrochem Energy Rev 2019, 2: 1-28.

[2] Wang S, Yang Y, Dong Y, et al. Recent progress in Ti-based nanocomposite anodes for lithium ion batteries. $J A d v$ Ceram 2019, 8: 1-18.

[3] Rehman WU, Wang HF, Manj RZA, et al. When silicon materials meet natural sources: Opportunities and challenges for low-cost lithium storage. Small 2019, 17: 1904508.

[4] Wang Q, Mao B, Stoliarov SI, et al. A review of lithium ion battery failure mechanisms and fire prevention strategies. Prog Energy Combust Sci 2019, 73: 95-131.

[5] Wang F, Chen G, Zhang N, et al. Engineering of carbon and other protective coating layers for stabilizing silicon anode materials. Carbon Energy 2019, 1: 219-245.

[6] Tu W, Bai Z, Deng Z, et al. In-situ synthesized Si@C materials for the lithium ion battery: A mini review. Nanomaterials (Basel) 2019, 9: E432.

[7] Zhong Y, Wu P, Ge S, et al. An egg holders-inspired 
structure design for large-volume-change anodes with long cycle life. J Alloys Compd 2020, 816: 152497.

[8] Liu N, Wu H, McDowell MT, et al. A yolk-shell design for stabilized and scalable Li-ion battery alloy anodes. Nano Lett 2012, 12: 3315-3321.

[9] Zhang L, Rajagopalan R, Guo HP, et al. Lithium-ion batteries: A green and facile way to prepare granadilla-like siliconbased anode materials for Li-ion batteries. Adv Funct Mater 2016, 26: 468.

[10] Liu N, Lu Z, Zhao J, et al. A pomegranate-inspired nanoscale design for large-volume-change lithium battery anodes. Nat Nanotechnol 2014, 9: 187-192.

[11] Entwistle J, Rennie A, Patwardhan S. A review of magnesiothermic reduction of silica to porous silicon for lithium-ion battery applications and beyond. J Mater Chem A 2018, 6: 18344-18356.

[12] Cook JB, Kim HS, Lin TC, et al. Tuning porosity and surface area in mesoporous silicon for application in Li-ion battery electrodes. ACS Appl Mater Interfaces 2017, 9: 19063-19073.

[13] Shi L, Wang W, Wang A, et al. Understanding the impact mechanism of the thermal effect on the porous silicon anode material preparation via magnesiothermic reduction. J Alloys Compd 2016, 661: 27-37.

[14] Guo S, Hu X, Hou Y, et al. Tunable synthesis of yolk-shell porous Silicon@Carbon for optimizing Si/C-based anode of lithium-ion batteries. ACS Appl Mater Interfaces 2017, 9: 42084-42092.

[15] Lin N, Han Y, Wang L, et al. Preparation of nanocrystalline silicon from $\mathrm{SiCl}_{4}$ at $200{ }^{\circ} \mathrm{C}$ in molten salt for high-performance anodes for lithium ion batteries. Angewandte Chemie Int Ed 2015, 54: 3822-3825.

[16] Lin N, Han Y, Zhou J, et al. A low temperature molten salt process for aluminothermic reduction of silicon oxides to crystalline Si for Li-ion batteries. Energy Environ Sci 2015, 8: 3187-3191.

[17] Weng Y, Chen G, Dou F, et al. In-situ growth of silicon carbide interface enhances the long life and high power of the mulberry-like Si-based anode for lithium-ion batteries. Energy Storage 2020, 32: 101856.

[18] Wang W, Wang Y, Gu L, et al. SiC@Si core-shell nanowires on carbon paper as a hybrid anode for lithium-ion batteries. J Power Sources 2015, 293: 492-497.

[19] Ngo DT, Le HTT, Pham XM, et al. Facile synthesis of $\mathrm{Si} @ \mathrm{SiC}$ composite as an anode material for lithium-ion batteries. ACS Appl Mater Interfaces 2017, 9: 32790-32800.

[20] Yu ZJ, Lv X, Lai SY, et al. ZrC-ZrB ${ }_{2}-\mathrm{SiC}$ ceramic nanocomposites derived from a novel single-source precursor with high ceramic yield. J Adv Ceram 2019, 8: 112-120.

[21] Chen Y, Zhang J, Chen X, et al. Facile preparation of Hollow $\mathrm{Si} / \mathrm{SiC} / \mathrm{C}$ yolk-shell anode by one-step magnesiothermic reduction. Ceram Int 2019, 45: 17040-17047.

[22] Wu P, Chen S, Liu A. The influence of contact engineering on silicon-based anode for li-ion batteries. Nano Sel 2021, 2:
$468-491$.

[23] Li L, Ding J, Xue JM. Macroporous silica hollow microspheres as nanoparticle collectors. Chem Mater 2009, 21: 3629-3637.

[24] Zhang H, Li X, Guo H, et al. Hollow $\mathrm{Si} / \mathrm{C}$ composite as anode material for high performance lithium-ion battery. Powder Technol 2016, 299: 178-184.

[25] Choi MJ, Xiao Y, Hwang JY, et al. Novel strategy to improve the Li-storage performance of micro silicon anodes. J Power Sources 2017, 348: 302-310.

[26] Kim B, Ahn J, Oh Y, et al. Highly porous carbon-coated silicon nanoparticles with canyon-like surfaces as a highperformance anode material for $\mathrm{Li}$-ion batteries. $J$ Mater Chem A 2018, 6: 3028-3037.

[27] Wu L, Zhou H, Yang J, et al. Carbon coated mesoporous Si anode prepared by a partial magnesiothermic reduction for lithium-ion batteries. J Alloys Compd 2017, 716: 204-209.

[28] Chen SQ, Shen LF, van Aken PA, et al. Dual-functionalized double carbon shells coated silicon nanoparticles for high performance lithium-ion batteries. Adv Mater 2017, 29: 1605650.

[29] Yu Z, Yang Y, Mao K, et al. Single-source-precursor synthesis and phase evolution of $\mathrm{SiC}-\mathrm{TaC}-\mathrm{C}$ ceramic nanocomposites containing core-shell structured TaC@C nanoparticles. $J$ Adv Ceram 2020, 9: 320-328.

[30] Luo L, Xu Y, Zhang H, et al. Comprehensive understanding of high polar polyacrylonitrile as an effective binder for Li-ion battery nano-Si anodes. ACS Appl Mater Interfaces 2016, 8: 8154-8161.

[31] Zhang JM, Tang JJ, Zhou XY, et al. Optimized porous Si/SiC composite spheres as high-performance anode material for lithium-ion batteries. ChemElectroChem 2019, 6: 450-455.

[32] Wang W, Wang Y, Gu L, et al. SiC@Si core-shell nanowires on carbon paper as a hybrid anode for lithium-ion batteries. J Power Sources 2015, 293: 492-497.

[33] Cho J. Porous Si anode materials for lithium rechargeable batteries. J Mater Chem 2010, 20: 4009-4014.

[34] Nie P, Liu X, Fu R, et al. Mesoporous silicon anodes by using polybenzimidazole derived pyrrolic $\mathrm{N}$-enriched carbon toward high-energy Li-ion batteries. ACS Energy Lett 2017 , 2: 1279-1287.

[35] Ryu J, Hong D, Choi S, et al. Synthesis of ultrathin Si nanosheets from natural clays for lithium-ion battery anodes. ACS Nano 2016, 10: 2843-2851.

[36] Li Q, Jiang R, Dou Y, et al. Synthesis of mesoporous carbon spheres with a hierarchical pore structure for the electrochemical double-layer capacitor. Carbon 2011, 49: 1248-1257.

[37] Yamauchi Y, Suzuki N, Radhakrishnan L, et al. Breakthrough and future: Nanoscale controls of compositions, morphologies, and mesochannel orientations toward advanced mesoporous materials. Chem Rec 2009, 9: 321-339.

[38] Huang X, Ding Y, Li K, et al. Spontaneous formation of the conformal carbon nanolayer coated Si nanostructures as the stable anode for lithium-ion batteries from silica nanomaterials. J Power Sources 2021, 496: 229833. 
[39] Jin Y, Tan Y, Hu X, et al. Scalable production of the silicon-tin Yin-Yang hybrid structure with graphene coating for high performance lithium-ion battery anodes. ACS Appl Mater Interfaces 2017, 9: 15388-15393.

[40] Chen Y, Hu Y, Shen Z, et al. Sandwich structure of grapheneprotected silicon/carbon nanofibers for lithium-ion battery anodes. Electrochimica Acta 2016, 210: 53-60.

[41] Parekh MH, Sediako AD, Naseri A, et al. In situ mechanistic elucidation of superior Si-C-graphite Li-ion battery anode formation with thermal safety aspects. Adv Energy Mater 2020, 10: 1902799.

[42] Yang Y, Lu Z, Xia J, et al. Crystalline and amorphous carbon double-modified silicon anode: Towards large-scale production and superior lithium storage performance. Chem Eng Sci 2021, 229: 116054.

[43] An Y, Tian Y, Wei H, et al. Porosity- and graphitizationcontrolled fabrication of nanoporous Silicon@Carbon for lithium storage and its conjugation with MXene for lithiummetal anode. Adv Funct Mater 2020, 30: 1908721.

[44] Cao L, Huang T, Cui M, et al. Facile and efficient fabrication of branched Si@C anode with superior electrochemical performance in LIBs. Small 2021, 17: 2005997.

Open Access This article is licensed under a Creative Commons Attribution 4.0 International License, which permits use, sharing, adaptation, distribution and reproduction in any medium or format, as long as you give appropriate credit to the original author(s) and the source, provide a link to the Creative Commons licence, and indicate if changes were made.

The images or other third party material in this article are included in the article's Creative Commons licence, unless indicated otherwise in a credit line to the material. If material is not included in the article's Creative Commons licence and your intended use is not permitted by statutory regulation or exceeds the permitted use, you will need to obtain permission directly from the copyright holder.

To view a copy of this licence, visit http://creativecommons. org/licenses/by/4.0/. 